\title{
A Note on Sturm-Liouville Problem for Difference Equations
}

\author{
Erdal Bas ${ }^{1, *}$, Ramazan Ozarslan ${ }^{2}$ \\ ${ }^{1,2}$ Firat University, Department of Mathematics, Elazig, Turkey
}

Abstract. In this study, Sturm-Liouville delay difference equation is considered with initial conditions as follows

$$
\begin{gathered}
-\Delta^{2} x(n-1)+q(n) x(n-k)=\lambda x(n), \\
x(1)=x(2)=1,
\end{gathered}
$$

where $k \in \mathbb{Z}^{+}, n$ is a finite integer. Sum representation is found and from here, behaviors of eigenfunctions are investigated for different $k$ values by illustrating with graphs and tables.

\section{Introduction}

Difference equations is discrete analogue of differential equations. So, some special type differential equations' discrete counterparts are analyzed paralelly. Sturm-Liouville problems is one of these examples. Sturm-Liouville problem has an important role because of a lot of applications in mathematical physics. Sutrm-Liouville differential and also difference equations were studied by variable authors [1-6]. Oscillation criteria and asymptotic behaviours of delay difference equations were studied by [7-14].

While generally, Sturm-Liouville difference equations are handle with a constant potential function, we handle it as a variable in our studies $[3,4]$. For the first time, we consider Sturm-Liouville delay difference equation.

In this study, our aim is to investigate behaviors of eigenfunctions for Sturm-Liouville delay difference initial value problem, defined as follows

$$
\begin{gathered}
-\Delta^{2} x(n-1)+q(n) x(n-k)=\lambda x(n), \\
x(1)=x(2)=1,
\end{gathered}
$$

where $k \in \mathbb{Z}^{+}, n$ is a finite integer, $\Delta$ is the forward difference operator, $\lambda$ is the positive spectral parameter, $q(n)$ is a real valued potential function.

\footnotetext{
* Corresponding author: erdalmat@yahoo.com
} 


\section{Preliminaries}

Definition 1. [15] Casoratian is defined by

$$
w(n)=\left[\begin{array}{cccc}
x_{1}(n) & x_{2}(n) & \ldots & x_{r}(n) \\
x_{1}(n+1) & x_{2}(n+1) & \ldots & x_{r}(n+1) \\
\vdots & \vdots & \ddots & \vdots \\
x_{1}(n+r-1) & x_{2}(n+r-1) & \ldots & x_{r}(n+r-1)
\end{array}\right],
$$

where $\sum_{i=a}^{b-1} x(i) \Delta y(i)=[x(i) y(i)]_{a}^{b}-\sum_{i=a}^{b-1} \Delta x(i) y(i+1)$. are solution functions.

Theorem 1 [17] (Wronskian-Type Identity) Let $x$ and $y$ be a solutions of (1). Then,

$$
W[x, y](n)=[x(n) \Delta y(n-1)-y(n) \Delta x(n-1)]
$$

is a constant.

Theorem 2 [15] (Summation by parts) If $a<b$, then

$$
\sum_{i=a}^{b-1} x(i) \Delta y(i)=[x(i) y(i)]_{a}^{b}-\sum_{i=a}^{b-1} \Delta x(i) y(i+1) .
$$

Theorem 3 [15] If $y(n)$ is an indefinite sum of $x(n)$, then

$$
\sum_{i=a}^{b-1} y(i)=x(b)-x(a) \text {. }
$$

\section{Main Results}

In this study, our aim is to investigate behaviors of eigenfunctions for Sturm-Liouville initial value delay difference problem, defined as follows

$$
\begin{gathered}
-\Delta^{2} x(n-1)+q(n) x(n-k)=\lambda x(n), \\
x(1)=x(2)=1,
\end{gathered}
$$

where $k \in \mathbb{Z}^{+}, n$ is a finite integer $\Delta$ is the forward difference operator, $\lambda$ is the positive spectral parameter, $q(n)$ is a real valued potential function.

Theorem 4 The representation of solution of Sturm-Liouville initial value delay difference problem (1)-(2) is found as follows;

$$
\begin{aligned}
x(n)=\frac{\sin 2 \theta-\sin \theta(1+q(1) x(1-k))}{\sin \theta} & \cos n \theta+\frac{-\cos 2 \theta+\cos \theta(1+q(1) x(1-k))}{\sin \theta} \sin n \theta \\
& -\frac{1}{\sin \theta} \sum_{i=1}^{n-1} q(i) x(i-k) \sin (n-i) \theta,
\end{aligned}
$$

where $\lambda=2-2 \cos \theta, \theta \neq k \pi, \sum_{i=a}^{b} .=0$, if $a>b$. 
Proof It is straightforward to obtain the representation of solution by variation of parameters method $[15,16]$. It is seen that the representation of solution holds for the problem (1)-(2) by writing into place.

\subsection{Applications}

Application 1 Let $q(n)=\frac{1}{n^{3 / 2}}, \lambda=1$, and corresponding eigenfunctions for $k=0$ and $k=1$ are $x_{1}(n)$ and $x_{2}(n)$ respectively, then we can obtain datas following

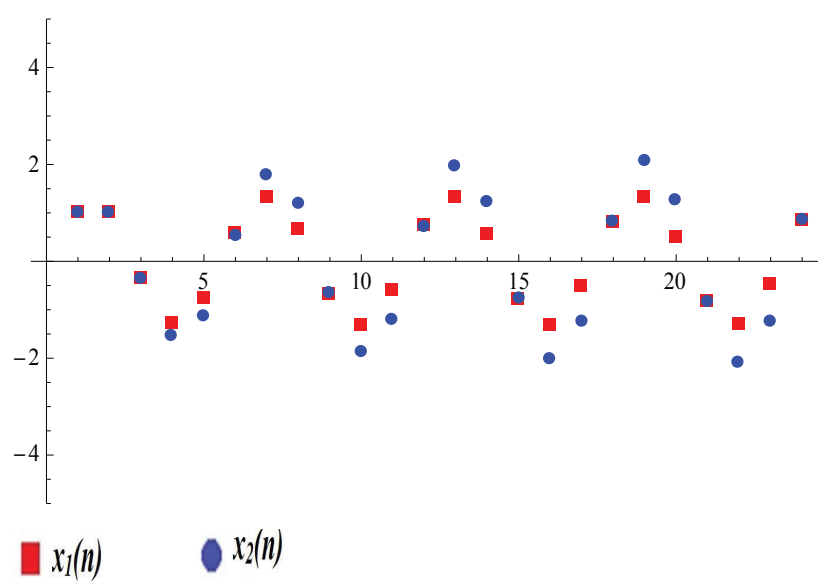

Fig. 1. Comparison of datas in Table 1

Table 1

\begin{tabular}{|c|c|c|}
\hline$n$ & $x_{1}(n)$ & $x_{2}(n)$ \\
\hline 1 & 1 & 1 \\
\hline 2 & 1 & 1 \\
\hline 3 & -0.35 & -0.35 \\
\hline 4 & -1.28 & -1.54 \\
\hline 5 & -0.77 & -1.14 \\
\hline 10 & -1.31 & -1.88 \\
\hline 11 & -0.59 & -1.22 \\
\hline 22 & 1.3 & -2.1 \\
\hline 23 & -0.46 & -1.25 \\
\hline 24 & 0.84 & 0.86 \\
\hline
\end{tabular}


Application 2 Let $q(n)=\frac{1}{n^{3 / 2}}, \lambda=1$, and corresponding eigenfunctions are $x_{1}(n)$ and $x_{2}(n)$ for $k=0$ and $k=2$ respectively, then we can obtain datas following

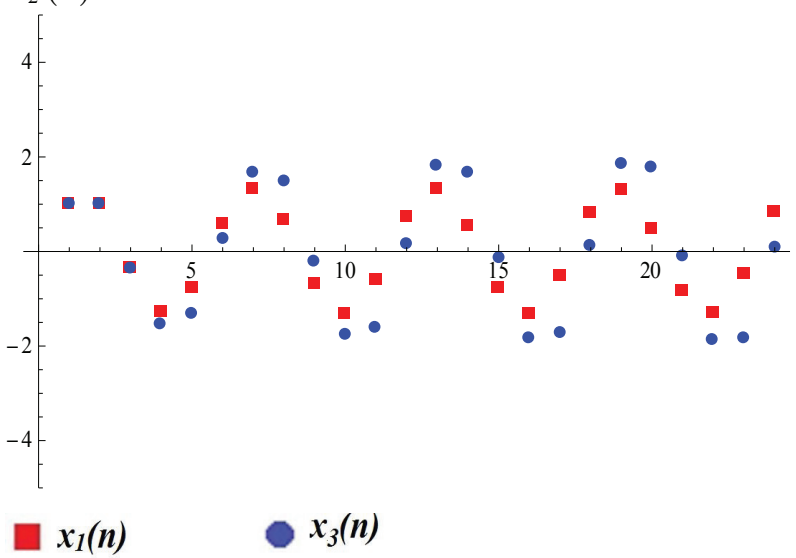

Fig. 2. Comparison of datas in Table 2

Table 2

\begin{tabular}{|c|c|c|}
\hline$n$ & $x_{1}(n)$ & $x_{3}(n)$ \\
\hline 1 & 1 & 1 \\
\hline 2 & 1 & 1 \\
\hline 3 & -0.35 & -0.35 \\
\hline 4 & -1.28 & -1.54 \\
\hline 5 & -0.77 & -1.31 \\
\hline 10 & -1.31 & -1.75 \\
\hline 11 & -0.59 & -1.6 \\
\hline 22 & 1.3 & -1.88 \\
\hline 23 & -0.46 & -1.81 \\
\hline 24 & 0.84 & 0.06 \\
\hline
\end{tabular}




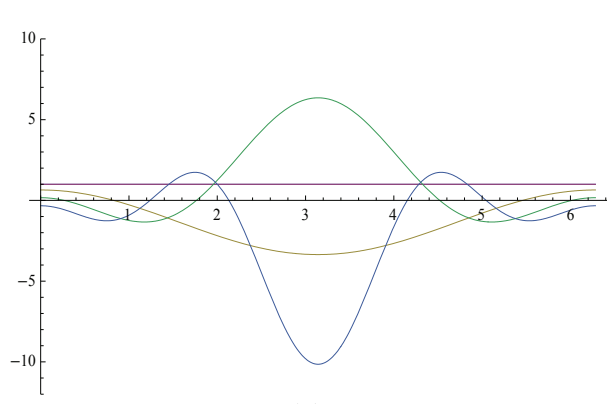

Fig. 3. $x_{1}(n), \mathrm{n}=1,2,3,4$

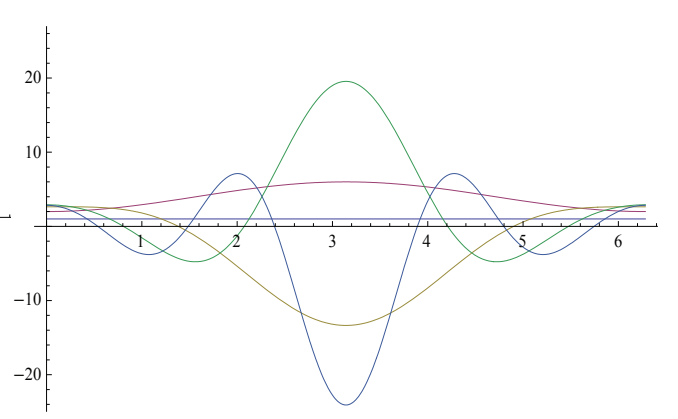

Fig.4. $x_{2}(n), \mathrm{n}=1,2, \ldots, 5$

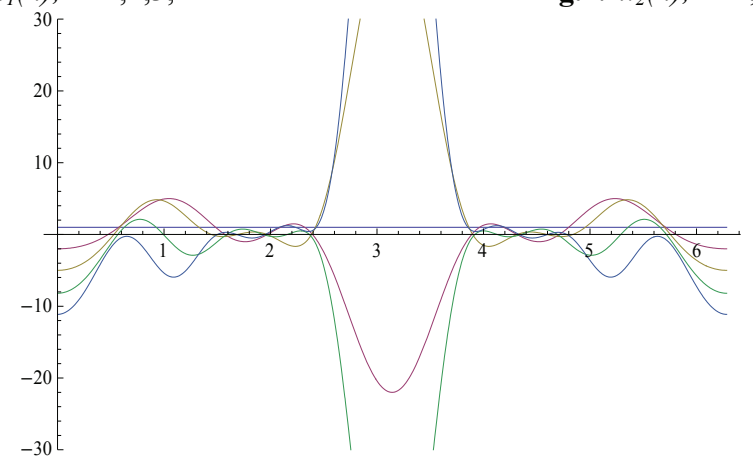

Fig. 5. $x_{3}(n), \mathrm{n}=1,2, \ldots, 5$

\section{Conclusion}

In this study, we investigated the behaviors of eigenfunctions for different $k$ values and obtained some numerical results. We showed the results by tables and figures. Obtained results show that the eigenfunctions are shifted for different $k$ values.

\section{References}

1. E. Bas, and E. Panakhov. "A New Approximation For Singular Inverse SturmLiouville Problem." Thai J. Math., 10.3 (2012): 685-692.

2. E. Bas, and F. Metin. "Fractional singular Sturm-Liouville operator for Coulomb potential." Adv. Difference Equ. ,2013.1 (2013): 300.

3. E. Bas, and R. Ozarslan. "Sturm-Liouville Problem via Coulomb Type in Difference Equations." Filomat 31.4 (2017): 989-998.

4. E. Bas, R. Ozarslan, Spectral results of Sturm-Liouville difference equation with Dirichlet boundary conditions: ICANAS 2016. AIP Publishing; 2016. p. 020065.

5. B. M. Levitan, I. S. Sargsjan, Introduction to Spectral Theory: Selfadjoint Ordinary Differential Operators, American Mathematical Society, Providence Rhode Island, p.5-8, 1975.

6. R. S. Hilscher, Spectral and oscillation theory for general second order Sturm-Liouville di_erence equations, Adv. Difference Equ. 82 (2012). 
7. I. Gyori, and M. Pituk. "Asymptotic Formulae for the Solutions of a Linear Delay Difference Equation-." J. Math. Anal. Appl. 195.2 (1995): 376-392.

8. Ch G. Philosand, I. K. Purnaras. "On linear Volterra difference equations with infinite delay.", Adv. Difference Equ. 2006.1 (2006): 1-28.

9. H. Péics, and A. Roznjik. "Asymptotic Behavior of Solutions of a Scalar Delay Difference Equations with Continuous Time." Novi Sad J. Math 38.3 (2008): 47-54.

10. R. D. Driver, G. Ladas, and P. N. Vlahos. "Asymptotic behavior of a linear delay difference equation." Proceedings of the American Mathematical Society (1992): 105112.

11. B. Karpuz, "Some oscillation and nonoscillation criteria for neutral delay difference equations with positive and negative coefficients." Comput. Math. Appl. 57.4 (2009): 633-642.

12. M. F. Aktaş, A. Tiryaki, and A. Zafer. "Oscillation of third-order nonlinear delay difference equations." Turkish J. Math. 36.3 (2012): 422-436.

13. W. Peiguang, and W. Wang. "Anti-periodic boundary value problem for first order impulsive delay difference equations.", Adv. Difference Equ. 2015.1 (2015): 93.

14. R. P. Agarwal, M. Bohner, S. R. Grace, D.O'Regan, (2005). Discrete Oscillation Theory, Hindawi Publ. Corporation, NewYork.

15. W.G. Kelley, A.C. Peterson, Difference Equations: An Introduction with Applications, Academic Press, San Diego (2001).

16. C.M. Bender, S.A. Orszag, Advanced Mathematical Methods for Scientists and Engineers: Asymptotic Methods and Perturbation Theory, Springer-Verlag, Newyork (1999).

17. A. Jirari, Second Order Sturm-Liouville Difference Equations and Orthogonal Polynomials. Memoirs of the American Mathematical Society, Vol. 113, Number 542, Providence Rhode Island; 1995. 